\title{
THE EFFECTS OF SPUTTER PRESSURE ON THE MICROSTRUCTURE OF CONi/Pt MULTILAYERS
}

\author{
M.D. Bijker, D.M. Donnet and J.C. Lodder \\ MESA Research Institute, University of Twente \\ P.O. Box 217, 7500 AE, Enschede, The Netherlands
}

\begin{abstract}
The change in microstructure in sputtered $\mathrm{CoNi} / \mathrm{Pt}$ Multilayers is studied as a function of the argon pressure. It is shown that as the sputter gas pressure increases the average grain size approximately stays constant, but the grains become more separated, the argon content decreases and the films tend to grow with rougher interfaces. Furthermore the increase in argon pressure also seems to lead to an increase in interface anisotropy.
\end{abstract}

KEYWORDS: multilayers, microstructure, TEM, XRD, VSM, magnetic anisotropy, XRF, magneto-optic media.

\section{INTRODUCTION}

Multilayer films exhibiting sufficiently large magnetooptical (M.O.) Kerr effects have drawn considerable attention and are considered as good candidates for future high density M.O. recording applications [1-3]. The multilayer's properties depend largely on the growth conditions and to study this relationship in some detail we have investigated the changes found in the microstructure as a function of the Ar pressure used during sputtering. Therefore a series of five $\mathrm{Co}_{50} \mathrm{Ni}_{50} / \mathrm{Pt}$ multilayers were prepared by sputtering: each film consists of a $\mathrm{Pt}$ seedlayer and a stack of $17 \mathrm{Co}_{50} \mathrm{Ni}_{50} / \mathrm{Pt}$ bilayers, sputtered at the same argon pressure. All relevant parameters of these samples are given in Table 1. Experimental details about the preparation of these layers are given elsewhere [4].

Table 1: Properties of the prepared samples.

\begin{tabular}{|c|c|c|c|c|c|}
\hline Sample & $\begin{array}{l}\mathrm{P}_{\mathrm{Ar}} \\
(\mu \mathrm{bar})\end{array}$ & $\begin{array}{l}\mathrm{t}_{\text {Seed }} \\
(\AA)\end{array}$ & $\begin{array}{l}\mathrm{t}_{\text {CoNi }} \\
(\AA)\end{array}$ & $\begin{array}{l}\mathrm{t}_{\mathrm{Pt}} \\
(\AA)\end{array}$ & $\begin{array}{l}\mathrm{K}_{\text {eff }} \\
\left(\mathrm{kJ} / \mathrm{m}^{3}\right)\end{array}$ \\
\hline $\mathrm{A}$ & 5 & 95 & 4.9 & 6.3 & -50 \\
\hline B & 8 & 95 & 5.0 & 5.8 & 7 \\
\hline C & 11 & 94 & 5.1 & 5.7 & 137 \\
\hline $\mathrm{D}$ & 30 & 95 & 8.0 & 6.1 & 324 \\
\hline E & 40 & 95 & 5.0 & 6.0 & 376 \\
\hline
\end{tabular}

\section{SAMPLE CHARACTERISATION}

Various measurements were performed in order to gain some inference on the effect that the sputtering process has on these multilayers. These included measurements of reflectivity, argon content, planar and cross sectional Transmission electron microscopy (TEM) and high angle $\mathrm{x}-$ ray diffraction (XRD).

$X$-ray fluorescence (XRF) measurements were performed on a Philips PW $1480 \times$-ray spectrometer (using a $\mathrm{Cr}$ tube) to measure the Ar content qualitatively. Reflectivity measurements were carried out using an UV 3101 PC Shimadzu UV-VIS-NIR scanning spectrophotometer at $\lambda=550 \mathrm{~nm}$. TEM planar and cross sectional views together with electron diffraction patterns were taken on a Philips CM 30 TEM operating at $300 \mathrm{kV}$. XRD was done with a Philips
PW 1820/00 $\mathrm{x}$-ray goniometer using $\mathrm{Cu} \mathrm{K}_{\alpha}$ radiation $(40 \mathrm{kV}, 30 \mathrm{~mA})$. In addition the hysteresis loops were measured using an Oxford Instruments VSM, model 3001 (sensitivity: $5 \mathrm{nAm}^{2}$, maximum field: $3 \mathrm{~T}$ ). The effective magnetic anisotropy was measured using a home build torque magnetometer (sensitivity: $4 \mathrm{nNm}$, maximum field: $1.8 \mathrm{~T})$

\section{MICROSTRUCTURAL CHARACTERISATION}

In order to investigate the texture of these multilayer samples, XRD was undertaken. A typical example of a $\theta-2 \theta$ scan is shown in figure 1. Here the result is shown of sample $\mathrm{D}$ with all the distinctive features indicated in the figure. These are the $\mathrm{Si}\langle 111\rangle$ substrate peak, the bilayer peak from the multilayer structure, the Pt seedlayer peak and a peak which is attributed to the multilayer stack. In figure 1 the latter is revered to as the $\mathrm{CoNi} / \mathrm{Pt}$ multilayer peak.

The fcc $\langle 111\rangle$ texture of the Pt seedlayer is identified by comparing the $\mathrm{Pt}$ seedlayer peak position to the ASTM diffraction file ( $\# 4$ - 802).

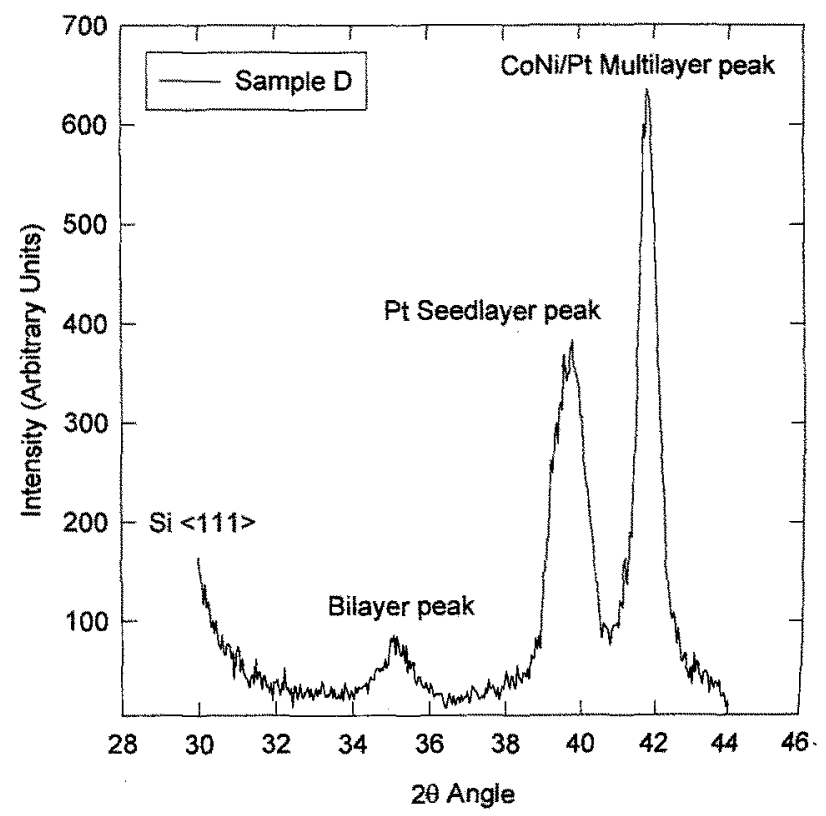

Figure 1: $\theta-2 \theta$ scan of sample D. 
The texture of the multilayer peak is determined by calculating a weighted average of the lattice spacing of the elements involved, assuming a fcc crystal structure [5]. This calculation makes use of the additional ASTM files \#4 - 850 (Ni) and \#15 - 806 (fcc Co) The fcc crystal structure was determined by comparing the phase diagrams of the $\mathrm{CoNi}$, CoPt and NiPt alloys [6]. Then the multilayer stack is found to have a pronounced fcc $\langle 111\rangle$ texture, thereby extending the texture of the $\mathrm{Pt}$ seedlayer. An indication of the multilayer peak position is also suggested by the peak positions from the CoPt (ASTM \#43 - 1358) and the $\mathrm{CoPt}_{3}$ (ASTM \#29 - 499) $\langle 111\rangle 2 \theta$ values respectively.

Since all the samples have approximately the same thickness, the intensity of the multilayer peak serves as a measure for the degree of crystal orientation and/or the grain size of the prepared samples. The peak intensity as a function of the argon pressure is plotted in figure 2 .

From this figure we conclude that the fcc texturing decreases with increasing argon pressure. To elucidate whether this is an effect of a decrease in grain size or the crystal orientation, planar TEM views were taken of all the samples mentioned in Table 1. The average grain size measured from about 50 grains per planar TEM view is also plotted in figure 2. From this we conclude that the fcc texture is decreased with increasing argon pressure, since the average grain size stays approximately constant.

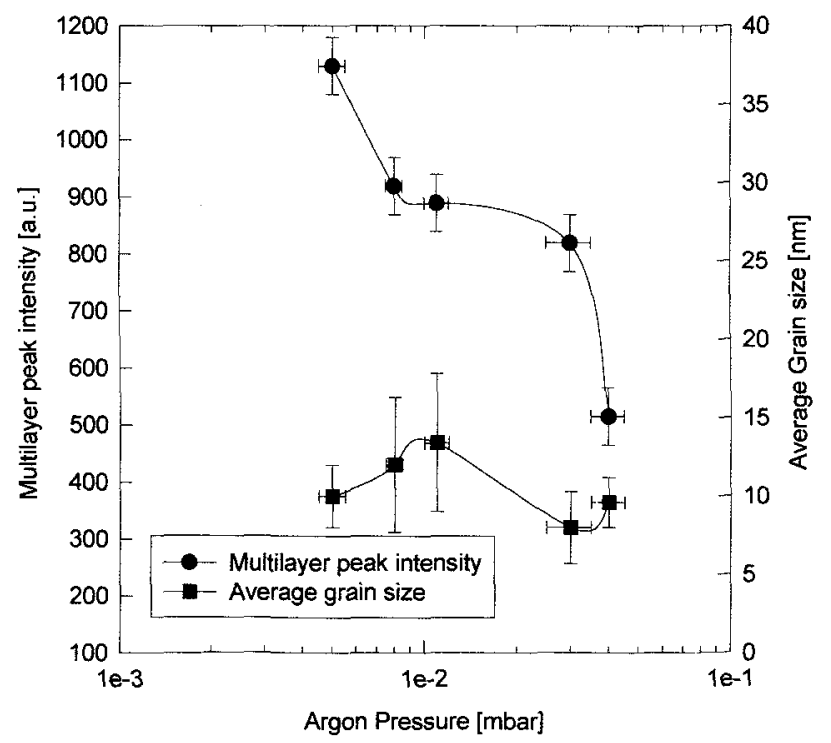

Figure 2: Multilayer peak intensity and average grain size as a function of the argon pressure. The drawn lines are to be used as a guide for the eye only.

Planar TEM views are presented in figure 3 for samples $A$ and $E$. The inset shows the electron diffraction pattern (ED), confirming the fcc $\langle 111\rangle$ texture. From the Si $\langle 111\rangle$ dots in the ED the cameralength was calibrated and used as an aid for labelling the diffraction rings of the polycrystalline multilayer stack.

In comparing figure $3 a$ and $3 b$ it is seen that the grains of sample $\mathrm{E}$ are considerably more isolated than in sample $\mathrm{A}$.
The planar views of the samples $B$ till $D$ are very similar to that of sample A. The isolated grains seem to appear only on the sample sputtered at $40 \mu \mathrm{bar}$.
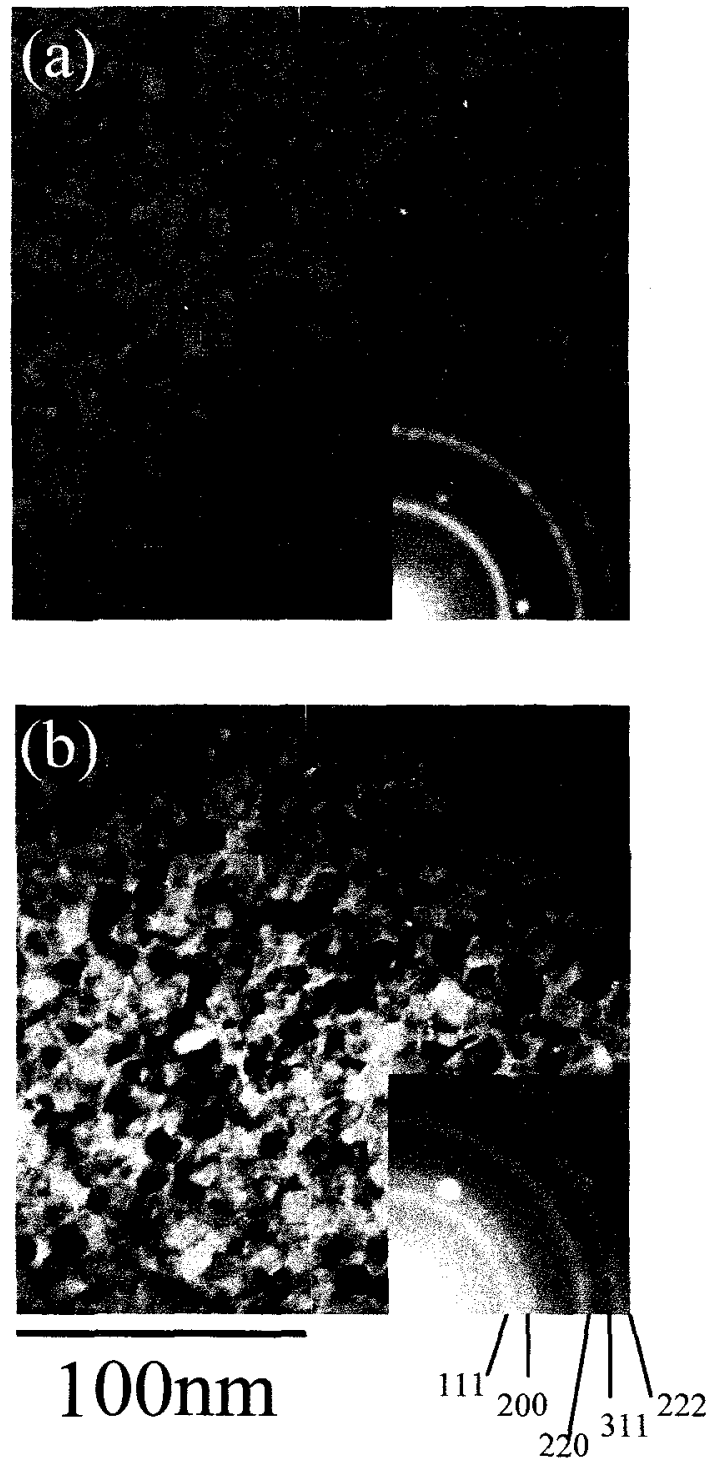

Figure $3 a \& 3 b$ : Planar TEM views of sample $A(a)$ and sample $E(b)$. On the inset the labelled electron diffraction patterns are shown.

To further investigate the effects of increased argon pressure, TEM cross sections were prepared of samples A and $\mathrm{D}$ and are presented in figure 4 . From this we see that in the low argon pressure regime the interfaces tend to be smooth, whereas in a higher sputter pressure regime the interfaces get rougher which probably leads to an increased surface roughness as well. Also figure 4 confirms the slightly thicker CoNi layers in sample D as with respect to the other samples mentioned in Table 1. 

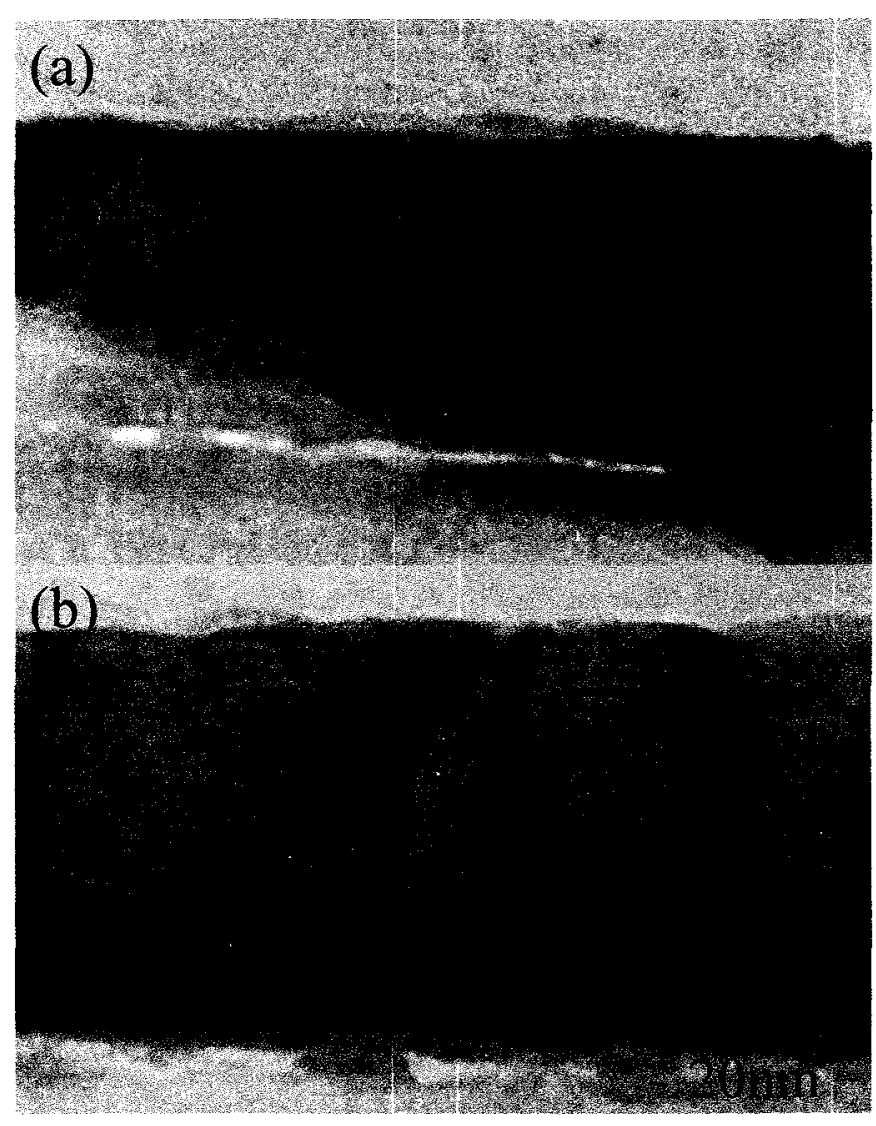

Figure $4 a \& 4 b$ : TEM Cross sections of sample A (a) and D (b).

To confirm the increasing surface roughness with increasing argon pressure, the coefficient of reflectivity was measured at $\lambda=550 \mathrm{~nm}$. These results are shown in figure 5 . Here we also measured the argon content of the layers in a qualitative way. As shown in figure 5 below, this content decreases with increasing pressure.

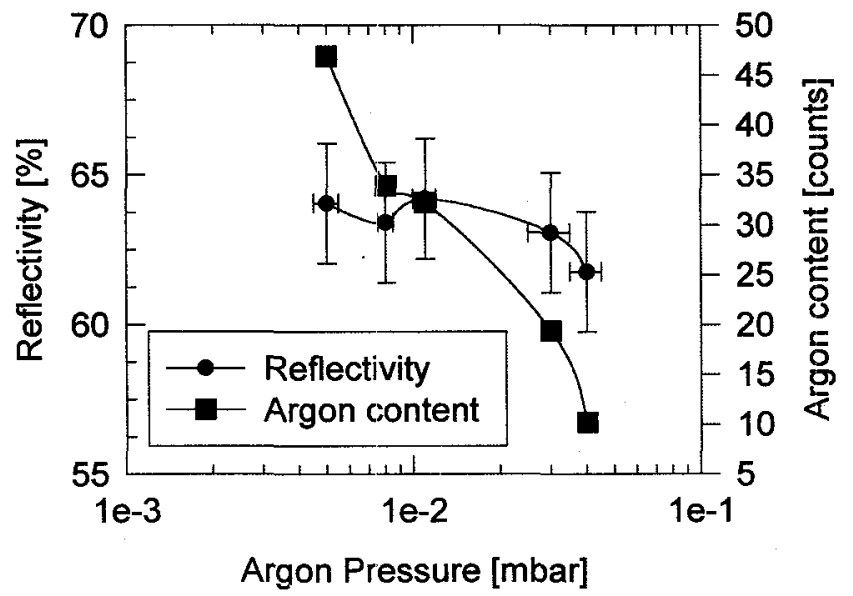

Figure 5: Reflection coefficient and qualitative argon content as a function of the argon pressure.

\section{MAGNETIC CHARACTERISATION}

The perpendicular coercivity of samples $B$ to $E$ were measured with both a VSM and a Kerr magnetometer and are shown in figure 6 . Also the effective magnetic anisotropy is shown in this figure. For convenience these values are also mentioned in Table 1. The saturation magnetisation of all the measured samples is $1060 \pm 60 \mathrm{kA} / \mathrm{m}$, based on the volume of the magnetic layers and not including polarisation effects of the platinum interlayers. This $\mathrm{M}_{\mathrm{s}}$ is slightly higher than expected for the bulk $\mathrm{Co}_{50} \mathrm{Ni}_{50}$ composition $(\sim 950 \mathrm{kA} / \mathrm{m})$.

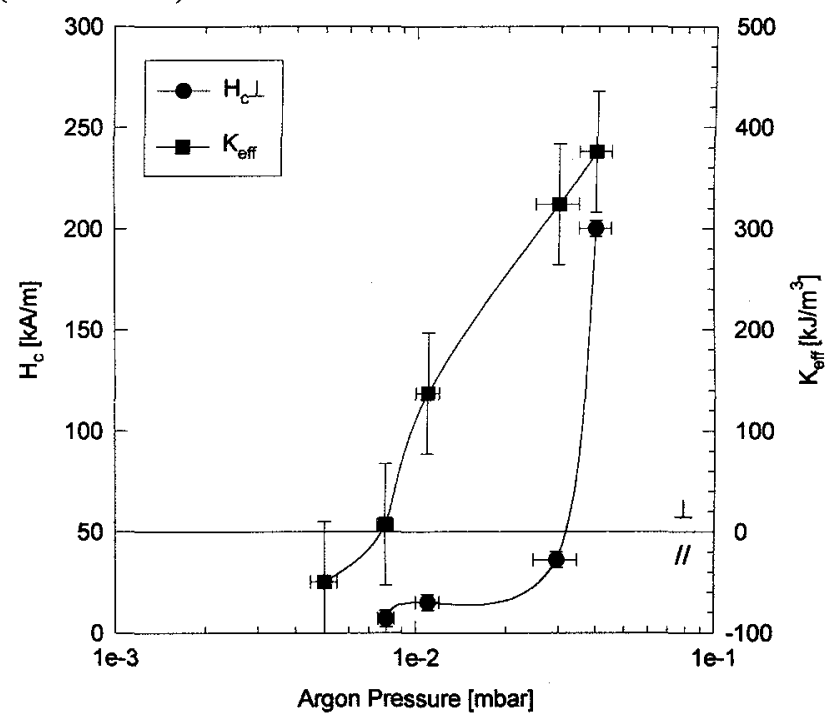

Figure 6: Perpendicular coercivity and effective magnetic anisotropy of samples $A$ to $E$ as a function of the argon pressure Note: Sample A has an in-plane coercivity and is therefore not shown.

From this figure it becomes clear that the effective magnetic anisotropy changes from in-plane to perpendicular at about $8 \mu \mathrm{bar}$. Furthermore the perpendicular coercivity gradually increases in the pressure range from 5 to $30 \mu \mathrm{bar}$ until it suddenly increases abruptly after $30 \mu$ bar to about five times its highest value in the total pressure range preceding this value.

\section{DISCUSSION}

From the jump in perpendicular coercivity after $30 \mu$ bar (fig. 6) and the isolated grain structure (fig. 3b) that seems to develop at the same pressure, it is estimated that there exists a reduced exchange coupling between the grains. However it is expected that there is still a magnetostatic coupling between adjacent grains. Furthermore as the argon pressure increases there seems to be a tendency for a transformation from a dense fibrous structure to a more porous one, accompanied by more tapered crystallites and separation by voids. These kinds of microstructural changes 
seem to correspond to the model that was developed by Thomton [7]. Although this model was derived for thicker films, this study seems to indicate a similar trend, judging from the figures 3 and 4.

As the argon pressure is increased, the oblique character of the deposition process also increases as the mean free path of the sputter gas species decreases and therefore the scattering of the sputtered neutrals will increase. Here the mean free path is reduced from $13.2 \mathrm{~mm}$ to $1.7 \mathrm{~mm}$ at $\mathrm{T}=300 \mathrm{~K}$ as the argon pressure is raised from 5 to 40 ubar. As expected from a similar investigation by Thornton et al. [8], the increased oblique particle flux is expected to be accompanied by a rougher film surface and is observed indirectly by a decrease in reflection coefficient as shown in figure 5 .

From this figure a decrease in argon content is also observed. This behaviour is expected to originate form the following principle [8-10]: Ar ions taking part in the plasma around the target can be neutralised upon reaching the target and reflected as high energetic neutral particles. These particles are thought to have sufficient excess energy to penetrate the growing film when reaching the substrate. Since the substrate to target distance is $100 \mathrm{~mm}$ in our sputtering system, the angle of reflection should be in the order of $180^{\circ}$ in order to be able to reach the substrate. In doing so, they have to diffuse through the gas column in between the target and substrate. On their way they are scattered by either neutral Ar atoms or sputtered target atoms. Therefore the process of energy transfer to the substrate is hindered to a greater extent at elevated $\mathrm{Ar}$ pressures and so less entrapped Ar atoms are expected at higher argon pressures.

From figure 6 it is observed that the perpendicular magnetic anisotropy (PMA) is increasing with increasing argon pressure. In identifying the possible anisotropy sources it is generally thought of as being a sum of a surface anisotropy and a volume anisotropy contribution [11]. The volume contribution on its own can be thought of as being the sum of magnetoelastic, magnetocrystalline and demagnetising components. From figure 6 it is seen that the anisotropy sources that force the easy axis (EA) in-plane are overcome by the anisotropy sources that force the EA to be perpendicular to the film surface.

The demagnetising volume anisotropy is able to reduce the PMA considerably because of the high $\mathrm{M}_{\mathrm{s}}$ values. From [12] we know that the magnetoelastic anisotropy in these CoNi/Pt multilayers lowers the PMA. If we assume that the magnetocrystalline anisotropy of the $\mathrm{Co}_{50} \mathrm{Ni}_{50}$ alloy equals the bulk value, then from [13] a value of $K_{1}=-$ $108 \mathrm{~kJ} / \mathrm{m}^{3}$ is found. Therefore the magneto- crystalline anisotropy is also thought to lower the PMA. However the implicit assumption made here is that this value will be the same for the whole range of argon pressures used in this study. From e.g. [2, Table II] in sputtered $\mathrm{Co} / \mathrm{Pd}$ multilayers it was found that raising the sputter pressure by approximately a factor of four, a decrease in both the interface anisotropy $\mathrm{K}_{\mathrm{s}}$ and the volume anisotropy $\mathrm{K}_{\mathrm{V}}$ of about $20 \%$ was observed. Therefore it is concluded that the total volume contribution to the magnetic anisotropy only decreases the PMA and therefore, since this opposes the observed trend in the PMA (fig. 6), it is concluded that increasing the argon pressure leads to an increase in the interface anisotropy of the films studied here.

\section{CONCLUSIONS}

From the microstructural characterisation of the CoNi/Pt multilayers in this study, it is found that to some extend the Thornton model could be validated. The isolated grain structure that evolved from sputtering at higher pressures leads to an abrupt increase in perpendicular coercivity and a gradual increase in effective magnetic anisotropy. Furthermore the increase in argon pressure also seems to lead to an increase in interface anisotropy.

\section{ACKNOWLEDGEMENTS}

The authors would like to thank Louise Vrielink and Marc Haast for carrying out the XRF and Kerr measurements respectively and Torsten Otto (University of Kassel, Germany) for confirming the VSM measurements.

\section{REFERENCES}

[1] S. Hashimoto and Y.Ochiai, J. Magn. Magn. Mater., $88(1990)$, p. 211.

[2] S. Hashimoto, Y. Ochiai, K Aso, J. Appl Phys, 66 (1989), p. 4909.

[3] W.B. Zeper, F.J.A.M. Greinadus, P.F. Carcia, IEEE Trans. Mag., 25 (1989), p. 3764.

[4] M.D. Bijker, W.P. Van Drent, J.C. Lodder, J, Magn. Magn. Mater., 48 (1995), p. 160.

[5] W.P. Van Drent, Ph.D. Thesis, University of Twente ISBN 90-9007985-8 (1995), p. 118.

[6] M. Hansen, Constitution of binary alloys, McGrawHill, (1958), p. 485,p. 492 \& p. 1031.

[7] J.A. Thornton, Ann. Rev. Mater. Sci., 7 (1977), p. 239.

[8] J.A. Thornton, D.W. Hoffman, Thin Solid Films, 171 (1989), p. 5.

[9] P.F.Carcia, S.I.Shah, W.B. Zeper, Appl. Phys. Lett, 56(23) (1990), p. 2345

[10] R.E. Somekh, J. Vac. Sci. Technol. A2 (1984), p. 1285

[11] H.J.G. Draaisma, W.J.M. de Jonge, F.J.A. den Broeder, J. Magn. Magn. Mater, 66 (1987), p. 351.

[12] W.P. Van Drent, M.D. Bijker, J.C. Lodder, Accepted for publication in J. Magn. Magn. Mater.

[13] R.M. Bozorth, Ferromagnetism, Princeton, N.J. (1951), p. 570 . 